\title{
Type I X-ray Bursts at Low Accretion Rates
}

\author{
Fang Peng*, Edward F. Brown ${ }^{\dagger}$ and James W. Truran*, ${ }^{* *}$ \\ ${ }^{*}$ Theoretical Astrophysics, California Institute of Technology, Pasadena, CA 91125 \\ ${ }^{\dagger}$ Department of Physics \& Astronomy, Michigan State University, East Lansing, MI 48824 \\ ${ }^{* *}$ Department of Astronomy \& Astrophysics, University of Chicago, Chicago, IL 60637 \\ ${ }^{\ddagger}$ Physics Division, Argonne National Laboratory, Argonne, IL 60439
}

\begin{abstract}
Neutron stars, with their strong surface gravity, have interestingly short timescales for the sedimentation of heavy elements. Recent observations of unstable thermonuclear burning (observed as X-ray bursts) on the surfaces of slowly accreting neutron stars $(<0.01$ of the Eddington rate) motivate us to examine how sedimentation of CNO isotopes affects the ignition of these bursts. We further estimate the burst development using a simple one-zone model with a full reaction network. We report a region of mass accretion rates for weak $\mathrm{H}$ flashes. Such flashes can lead to a large reservoir of $\mathrm{He}$, the unstable burning of which may explain some observed long bursts (duration $\sim 1000 \mathrm{~s})$.
\end{abstract}

Keywords: diffusion - stars: neutron $-\mathrm{X}$-rays: binaries $-\mathrm{X}$-rays: bursts

PACS: 97.60.Jd, 98.70.Qy

\section{INTRODUCTION}

An ionized plasma in a gravitational field develops an electric field sufficient to levitate the ions and ensure overall charge neutrality. When there is more than one species of ion present, the ions will experience a differential force: lighter ions float upward (defined by the local gravitational field) and heavier ions sink downward.

Accreting neutron stars, with their strong surface gravity $\approx 2.0 \times 10^{14} \mathrm{~cm} \mathrm{~s}^{-2}$, are an ideal place to look for the effects of sedimentation. The sedimentation of heavy elements and resulting nucleosynthesis in the envelope of isolated neutron stars cooling from birth was first described by Rosen $[1,2]$ and has been studied in detail by Chang and Bildsten [3, 4]. For accreting neutron stars, the rapid stratification removes heavy nuclei from the photosphere for accretion rates $\dot{M} \lesssim 10^{-12} M_{\odot} \mathrm{yr}^{-1}$ [5]. Deeper in the neutron star envelope, the differentiation of the isotopes can alter the nuclear burning, namely unstable $\mathrm{H} / \mathrm{He}$ burning and rapid proton-capture process (rp-process), that powers Type I X-ray bursts.

Recent long-term monitoring of the galactic center with BeppoSAX led to the discovery of nine "burst-only sources" [see 6, and references therein]. These sources did not have persistent fluxes detectable with the BeppoSAX/WFC, thus must have extremely low accretion luminosities $\left(L_{X}<10^{36} \mathrm{ergs} \mathrm{s}^{-1} \simeq 0.01 L_{\text {Edd }}\right)$. If the accretion is not concentrated onto a small surface area, so that the local accretion rate is $\dot{m} \approx \dot{M} /\left(4 \pi R^{2}\right)$, then the sedimentation timescale for $\mathrm{CNO}$ nuclei, defined as the time required to move a scale height relative to the center of mass of a fluid element, is less than the accretion timescale, defined as the time for a fluid element to reach a given depth. An estimate of this accretion rate was noted earlier by Wallace et al. [7]. As a result, sedimentation in (C) 2007 American Institute of Physics 978-0-7354-0434-2/07/\$23.00 
the accreted envelope must be considered in treating the unstable ignition of hydrogen and helium for these low- $\dot{m}$ sources. At somewhat higher accretion rates, $L_{X} \sim 0.01 L_{\mathrm{Edd}}$, several bursts have been observed with longer durations, $\lesssim 1000 \mathrm{~s}$, that are intermediate between mixed $\mathrm{H} / \mathrm{He}$ bursts and superbursts.

Motivated by these observations, we explore the unstable ignition of hydrogen and helium at low accretion rates and pay particular attention to the regime where the mass accretion rate is less than the critical rate needed for stable $\mathrm{H}$ burning [8]. We also study how the sedimentation of $\mathrm{CNO}$ nuclei affects the unstable ignition of $\mathrm{H}$ and $\mathrm{He}$ in an accreted neutron star envelope. For a full reporting of these results, please see [9].

\section{THE ROLE OF SEDIMENTATION ON IGNITION CONDITIONS}

To study the accumulation of $\mathrm{H}$ and $\mathrm{He}$, we construct models of a plane-parallel atmosphere in hydrostatic equilibrium, and for simplicity we neglect the terms coupling thermal and particle diffusion [see 10, and references therein]. We follow the treatment of Burgers [11] and construct for each species an equation of continuity and momentum conservation. Collisions are described by a resistance coefficient $K_{s t}=n_{s} n_{t}\left\langle\sigma_{s t} v_{s t}\right\rangle$, with $v_{s t}$ the center-of-mass relative velocity between particles of types $s$ and $t$ and $\sigma_{s t}$ the cross-section between these particles.

As an illustration of how sedimentation changes the structure of the envelope, consider a trace species, labeled 2 , in a background of a species labeled 1, i.e., $n_{1} \gg n_{2}$ and species 1 has velocity $w_{1}=0$. The electric force is $e E=m_{\mathrm{u}} g A_{1} /\left(Z_{1}+1\right)$ where the electrons are non-degenerate and $e E=m_{\mathrm{u}} g A_{1} / Z_{1}$ where the electrons are degenerate. In these expressions, $m_{\mathrm{u}}$ is the atomic mass unit. Substituting $E$ into the equation of motion for species 2 then determines the sedimentation velocity, $w_{\text {sed }}=w_{2}=n_{2}\left(A_{2} m_{\mathrm{u}} g-\right.$ $\left.Z_{2} e E\right) / K_{12}$. We chose the sign of $w_{\text {sed }}$ to be positive if species 2 moves downward.

Using the Stokes-Einstein relation to determine $K_{12}$ from the drag coefficient for a liquid sphere ([12]), and a nonrelativistic electron equation of state, we find the sedimentation velocity of a trace nucleus [see 13]

$$
w_{\text {sed }}=2 \times 10^{-3} g_{14} \frac{T_{7}^{0.3}}{\rho_{5}^{0.6}} \frac{\left(A_{2} Z_{1}-Z_{2} A_{1}\right) A_{1}^{0.1}}{Z_{1}^{2.3} Z_{2}^{0.3}} \mathrm{cms}^{-1},
$$

where we use the common shorthand $g_{14}=g /\left(10^{14} \mathrm{cms} \mathrm{s}^{-2}\right), T_{7}=T /\left(10^{7} \mathrm{~K}\right)$, and $\rho_{5}=\rho /\left(10^{5} \mathrm{~g} \mathrm{~cm}^{-3}\right)$. In a pure $\mathrm{H}$ plasma, the sedimentation velocity ${ }^{1}$ is greater than the mean velocity $u=\dot{m} / \rho$ for $\dot{m}<400 \mathrm{~g} \mathrm{~cm}^{-2} \mathrm{~s}^{-1} T_{7}^{0.3} \rho_{5}^{0.4}\left(A_{2}-Z_{2}\right) / Z_{2}^{0.3}$; under conditions at which $\mathrm{H}$ ignites $\left(T_{7} \sim 5 ; \rho_{5} \sim 4\right)$ this corresponds to $\dot{m}<0.02 \dot{m}_{\text {Edd }}$ and $\dot{m}<0.05 \dot{m}_{\text {Edd }}$ for a trace ${ }^{4} \mathrm{He}$ and ${ }^{12} \mathrm{C}$ nucleus, respectively. Here $\dot{m}_{\mathrm{Edd}}=2 m_{\mathrm{p}} c /\left[\left(1+X_{\mathrm{H}}\right) \sigma_{\mathrm{TH}} R\right]=$ $8.8 \times 10^{4} \mathrm{~g} \mathrm{~cm}^{-2} \mathrm{~s}^{-1}$ is the local Eddington mass accretion rate for a solar composition, $\sigma_{\mathrm{TH}}$ is the Thomson scattering cross section, and $X_{\mathrm{H}}$ is the hydrogen mass fraction.

We numerically solve for the evolution of the accreting envelope prior to unstable ignition by coupling the diffusion equations to the equations for temperature $T$ and heat

\footnotetext{
${ }^{1}$ Throughout this paper we use a Newtonian metric and assume a neutron star mass $1.4 M_{\odot}$ and radius $10 \mathrm{~km}$, so that $g_{14}=1.9$.
} 
flux $F$,

$$
\frac{\partial T}{\partial y}=\frac{F}{\rho K}, \quad \frac{\partial F}{\partial y}=C_{P}\left(\frac{\partial T}{\partial t}+\dot{m} \frac{\partial T}{\partial y}\right)-\frac{C_{P} T \dot{m}}{y} \nabla_{\mathrm{ad}}-\varepsilon_{\mathrm{nucl}},
$$

where $d y=-\rho d z$ is the column density, $C_{P}$ is the specific heat at constant pressure, $\varepsilon_{\text {nucl }}$ is the nuclear heating rate per unit mass, and $\nabla_{\mathrm{ad}} \equiv(\partial \ln T / \partial \ln P)_{S}$. For detailed description of the numerical method in solving these equations, please refer to [9].

We take the composition of matter accreted from the companion star to be roughly solar composition in ${ }^{1} \mathrm{H}$ and ${ }^{4} \mathrm{He}$, and distribute the remaining mass evenly between ${ }^{12} \mathrm{C}$ and ${ }^{16} \mathrm{O}: X\left({ }^{1} \mathrm{H}\right)=0.71, X\left({ }^{4} \mathrm{He}\right)=0.27, X\left({ }^{12} \mathrm{C}\right)=0.01$, and $X\left({ }^{16} \mathrm{O}\right)=0.01$. Below the fuel layer are ashes from previous X-ray bursts; we set the ash composition to ${ }^{64} \mathrm{Zn}$, consistent with the findings of recent one-dimensional calculations of repeated X-ray bursts $[14,15]$. We use a radiative-zero outer boundary, and for the inner boundary, at the bottom of the ash layer, we set the flux to a constant value, $F_{\mathrm{c}}=0.1 \mathrm{MeV} \dot{m} / m_{\mathrm{u}}[16]$. This flux is set by reactions in the deep crust-electron captures, neutron emissions, and pycnonuclear reactions.

We explore the evolution of the accreting neutron star envelope for accretion rates $10^{-4}-0.6 \dot{m}_{\text {Edd }}\left(10-5 \times 10^{4} \mathrm{~g} \mathrm{~cm}^{-2} \mathrm{~s}^{-1}\right.$ for accretion of a solar composition mixture). For each accretion rate, we ran calculations both with and without isotopic sedimentation, and we follow the thermal and chemical evolution of the mixture until the envelope becomes thermally unstable. We determine this ignition point using a simple one-zone criterion, $\left(\partial \varepsilon_{\text {nucl }} / \partial T\right)_{P}>\left(\partial \varepsilon_{\text {cool }} / \partial T\right)_{P}$, where $\varepsilon_{\text {cool }}=\rho K T / y^{2}$ is an approximation of the local cooling rate. The mass fraction of $\mathrm{H}$ and $\mathrm{He}$ at the column where either $\mathrm{H}$ or He unstably ignites is plotted in fig. 1, as a function of mass accretion rates. When sedimentation is included, the abundance of $\mathrm{H}$ at the base of the accreted envelope is depressed. It is tantalizing that the accretion rate at which mixed $\mathrm{H} / \mathrm{He}$ ignition occurs is increased by a factor of 2 when sedimentation is taken into account, and we speculate that this might alleviate the discrepancy between the predicted transition in burst duration [8] and recent observations [see, for example 17]

\section{BURSTS AT LOW ACCRETION RATES}

When the temperature in the neutron star envelope is sufficiently low, the CNO cycle becomes temperature dependent; as a result, the ignition of $\mathrm{H}$ becomes thermally unstable at low accretion rates [8]. As a first investigation of the unstable ignition of $\mathrm{H}$ when the atmosphere is stratified, we perform several calculations of the burst nucleosynthesis. We approximate the cooling by a one-zone finite differencing over the envelope,

$$
C_{P} \frac{d T}{d t}=\varepsilon_{\mathrm{nucl}}-\varepsilon_{\mathrm{cool}},
$$

where $\varepsilon_{\text {cool }}=\rho K T y^{-2}$ and we evaluate $\varepsilon_{\text {nucl }}$ from a reaction network. Included in this network are 686 isotopes covering proton-rich nuclei up to $\mathrm{Xe}$ [see 18]. The reaction rates are taken from the compilation REACLIB [see 19, 20, and references therein], and consist of experimental rates and Hauser-Feshbach calculations with the code NONSMOKER [20]. The initial temperature, column density, and composition are taken from 


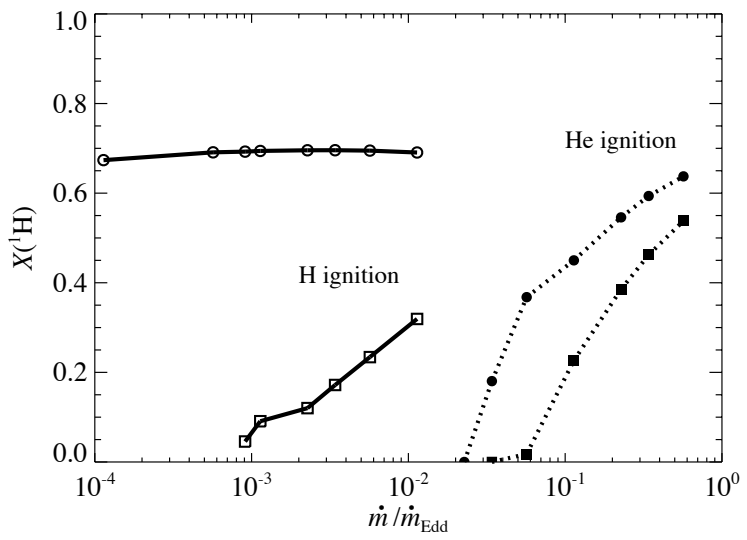

FIGURE 1. Mass fraction of hydrogen at the column where either $\mathrm{H}$ (open symbols, solid lines) or $\mathrm{He}$ (filled symbols, dotted lines) unstably ignites, as a function of mass accretion rates. We show results for which sedimentation is ignored (circles), and for which it is included (squares). From [9] and reproduced by permission of the AAS.

the values of the quasi-static calculation at the ignition point. For the unstable ignition of $\mathrm{H}$, the temperature in the accreted envelope is set by the flux emergent from the deeper ocean and crust. As a result, the accretion rate at which the burst behavior changes will depend on assumptions about the heating in the crust.

We shall first describe the outcome of these one-zone calculations (eq. [3]). The left panel of Figure 2 shows the post-ignition evolution for accretion rates $9.1 \times 10^{-4} \dot{m}_{\text {Edd }}$ $\left(80 \mathrm{~g} \mathrm{~cm}^{-2} \mathrm{~s}^{-1}\right.$; solid lines $), 1.1 \times 10^{-3} \dot{m}_{\mathrm{Edd}}\left(100 \mathrm{~g} \mathrm{~cm}^{-2} \mathrm{~s}^{-1} ;\right.$ dotted lines $)$, and $2.3 \times 10^{-3} \dot{m}_{\text {Edd }}\left(200 \mathrm{~g} \mathrm{~cm}^{-2} \mathrm{~s}^{-1}\right.$; dashed lines $)$. We plot three different quantities: the evolution of temperature (top panel), the heat flux, $F_{\text {cool }}=y \varepsilon_{\text {cool }}$, normalized to the accretion flux (middle panel), and the mass fractions of hydrogen and helium (bottom panel). We then repeat this calculation at higher mass accretion rates (Fig. 2, right panel), $5.7 \times 10^{-3} \dot{m}_{\text {Edd }}\left(500 \mathrm{~g} \mathrm{~cm}^{-2} \mathrm{~s}^{-1} ;\right.$ solid lines $)$ and $1.1 \times 10^{-2} \dot{m}_{\mathrm{Edd}}\left(10^{3} \mathrm{~g} \mathrm{~cm}^{-2} \mathrm{~s}^{-1} ;\right.$ dotted lines).

It is immediately clear that there are two very different outcomes for the $\mathrm{H}$ ignition. At lower accretion rates, the rise in temperature following $\mathrm{H}$ ignition is sufficient to trigger a vigorous He flash with a decay timescale $\sim 10 \mathrm{~s}$ (Fig. 2, middle-left panel). At higher accretion rates, however, the flash is too weak to ignite He. As an interpretation of these results, recall that the ignition curve for the $3 \alpha$ reaction has a turning point at $y_{\text {turn }} \approx 5 \times 10^{7} \mathrm{~g} \mathrm{~cm}^{-2}$, which terminates the unstable branch [21, 22]. At the higher accretion rates (Fig. 2, right panel) the ignition of $\mathrm{H}$ occurs at $y<y_{\text {turn }}$, and the local rise in temperature does not trigger unstable He ignition. In fact, the temperature rise is not even sufficient to initiate convection, as the radiative temperature gradient needed to carry $F_{\text {cool }}$ is $d \ln T / d \ln P \approx 0.25<(\partial \ln T / \partial \ln P)_{s}$. In contrast, at lower accretion rates (Fig. 2, left panel), the ignition of $\mathrm{H}$ occurs at $y>y_{\text {turn }}$, and the rise in temperature will ignite the triple- $\alpha$ reaction.

If subsequent $\mathrm{H}$ flashes do not ignite the underlying $\mathrm{He}$, then a large layer of nearly 

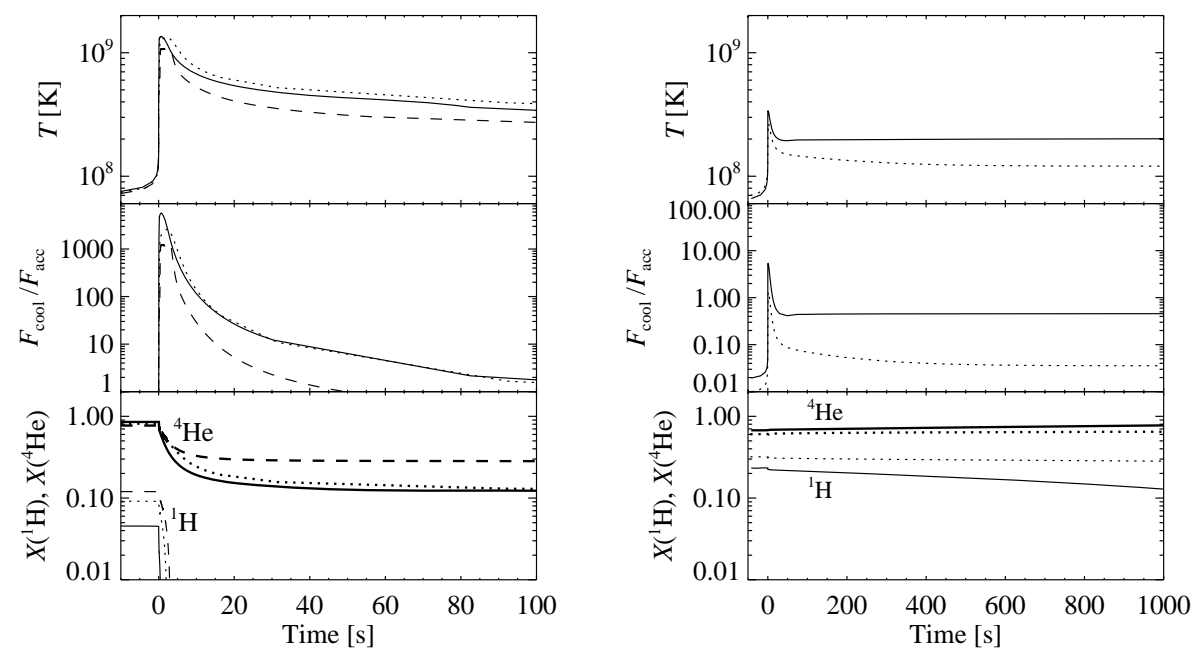

FIGURE 2. One-zone burst calculation following unstable $\mathrm{H}$ ignition. Left: for three mass accretion rates, $\dot{m} / \dot{m}_{\text {Edd }}=9.1 \times 10^{-4}$ (solid lines), $1.1 \times 10^{-3}$ (dotted lines) and $2.3 \times 10^{-3}$ (dashed lines), respectively. Right: for two mass accretion rates, $\dot{m} / \dot{m}_{\mathrm{Edd}}=5.7 \times 10^{-3}$ (solid lines) and 0.011 (dotted lines), respectively. Top panel: temperature evolution; Middle panel: the ratio of one-zone cooling flux to the accretion flux; Bottom panel: mass fraction of hydrogen (thin lines) and ${ }^{4} \mathrm{He}$ (thick lines), respectively. Diffusion and sedimentation is included. From [9], and reproduced by permission of the AAS.

pure He will accumulate. Because our system is not burning $\mathrm{H}$ steadily, the temperature in the He layer is colder than if the burning were in steady-state. As a result, a large He layer should accumulate. To get the thermal structure of an accumulated pure $\mathrm{He}$ layer, we integrated equations (2), with $\partial / \partial t \rightarrow 0$, from the column where $\mathrm{H}$ ignition occurred, and set the temperature there to that found in the accumulating model. For $\dot{m} / \dot{m}_{\text {Edd }}=5.7 \times 10^{-3}$ and 0.011 , the He ignition column density varies from $y \approx$ $2 \times 10^{9}-3 \times 10^{11} \mathrm{~g} \mathrm{~cm}^{-2}$ (recurrence time $\approx 0.06-19 \mathrm{yr}$ ) for $F_{\mathrm{c}} m_{\mathrm{u}} / \dot{\mathrm{m}}$ varies from 1.0 to 0.1 . Our one-zone approximation gives a cooling time $\tau \approx 400 \mathrm{~s}\left[y /\left(10^{10} \mathrm{~g} \mathrm{~cm}^{-2}\right)\right]$, which roughly agrees with the long burst duration.

\section{CONCLUSION}

Using a simplified numerical model of an accreting neutron star envelope that allows for differential isotopic velocities, we have undertaken a first study of the effects of sedimentation and diffusion on the unstable ignition of hydrogen and helium on the surfaces of accreting neutron stars, and have investigated the outcome of unstable hydrogen ignition using simple one-zone models. Our principal conclusions are:

1. The effect of sedimentation changes the conditions at $\mathrm{H} / \mathrm{He}$ ignition even for $\dot{m} \gtrsim 0.1 \dot{m}_{\text {Edd }}$.

2. There is a range of accretion rates for which unstable $\mathrm{H}$ ignition does not trigger 
a He flash. This range depends on the flux from the deep crustal heating and the degree of settling of $\mathrm{He}$ and $\mathrm{CNO}$ nuclei. We speculate that successive weak $\mathrm{H}$ flashes can lead to the accumulation of a large reservoir of $\mathrm{He}$; this may explain the long bursts observed from some sources.

\section{ACKNOWLEDGMENTS}

This work is supported in part by the Joint Institute for Nuclear Astrophysics under NSF-PFC grant PHY 02-16783, and by the Department of Energy under grant B523820 to the Center for Astrophysical Thermonuclear Flashes at the University of Chicago. EFB is supported by the NSF under grant AST-0507456; JWT acknowledges support from the U.S. DOE, under contract No. W-31-109-ENG-38.

\section{REFERENCES}

1. L. C. Rosen, $A p \& S S$ 5, 150 (1969).

2. L. C. Rosen, $A p \& S S$ 5, 92 (1969).

3. P. Chang, and L. Bildsten, ApJ 585, 464-474 (2003).

4. P. Chang, and L. Bildsten, ApJ 605, 830-839 (2004).

5. L. Bildsten, E. E. Salpeter, and I. Wasserman, ApJ 384, 143-176 (1992).

6. R. Cornelisse, J. J. M. in't Zand, E. Kuulkers, J. Heise, F. Verbunt, M. Cocchi, A. Bazzano, L. Natalucci, and P. Ubertini, Nuclear Physics B Proceedings Supplements 132, 518-523 (2004).

7. R. K. Wallace, S. E. Woosley, and T. A. Weaver, ApJ 258, 696 (1982).

8. M. Y. Fujimoto, T. Hanawa, and S. Miyaji, ApJ 247, 267-278 (1981).

9. F. Peng, E. F. Brown, and J. W. Truran, ApJ in press (2006), a stro-ph/ 0609583.

10. C. Paquette, C. Pelletier, G. Fontaine, and G. Michaud, ApJS 61, 177-195 (1986).

11. J. M. Burgers, Flow Equations for Composite Gases, Academic Press, Inc., New York, 1969.

12. L. Bildsten, and D. M. Hall, ApJ 549, 219 (2001).

13. E. F. Brown, L. Bildsten, and P. Chang, ApJ 574, 920-929 (2002).

14. S. E. Woosley, A. Heger, A. Cumming, R. D. Hoffman, J. Pruet, T. Rauscher, J. L. Fisker, H. Schatz, B. A. Brown, and M. Wiescher, ApJS 151, 75 (2004).

15. J. L. Fisker, E. Brown, M. Liebendoerfer, H. Schatz, and F. K. Thielemann, Nucl. Phys. A 758, 447 (2005).

16. E. F. Brown, ApJ 531, 988 (2000), astro-ph/9910215.

17. P. R. den Hartog, J. J. M. in't Zand, E. Kuulkers, R. Cornelisse, J. Heise, A. Bazzano, M. Cocchi, L. Natalucci, and P. Ubertini, A\&A 400, 633-641 (2003).

18. H. Schatz, A. Aprahamian, V. Barnard, L. Bildsten, A. Cumming, M. Ouellette, T. Rauscher, F.-K. Thielemann, and M. Wiescher, Phys. Rev. Lett. 86, 3471 (2001), astro-ph/0102418.

19. F. Thielemann, M. Arnould, and J. Truran, "Thermonuclear Reaction Rates from Statistical Model Calculations," in Advances in Nuclear Astrophysics, edited by E. Vangioni-Flam, J. Audouze, M. Casse, J.-P. Chieze, and J. Tran Thanh Van, Editions Frontières, Gif-sur-Yvette, 1986, p. 525, URL http: / /ie.lbl.gov/astro/friedel.html.

20. T. Rauscher, and F. Thielemann, At. Data Nucl. Data Tables 75, 1 (2000), astro-ph/ 0004059.

21. L. Bildsten, "Thermonuclear Burning on Rapidly Accreting Neutron Stars," in The Many Faces of Neutron Stars, edited by A. Alpar, R. Buccheri, and J. van Paradijs, NATO ASI ser. C, Kluwer, Dordrecht, 1998, vol. 515, p. 419.

22. A. Cumming, and L. Bildsten, ApJ 544, 453-474 (2000). 